\title{
A Dynamic Formation Procedure of Information Flow Networks
}

\author{
Hongwei GAO \\ College of Mathematics, Qingdao University, Qingdao 266071, China \\ E-mail:gaohongwei@qdu.edu.cn \\ Han QIAO* \\ Management School, University of Chinese Academy of Sciences, Beijing 100190, China \\ E-mail: qiaohan@ucas.ac.cn \\ Artem SEDAKOV \\ Faculty of Applied Mathematics and Control Processes, \\ Saint Petersburg State University, Saint Petersburg, 198504, Russia \\ E-mail:a.sedakov@spbu.ru \\ Lei WANG \\ Department of Mathematics, Teachers College of Qingdao University, Qingdao 266071, China \\ E-mail:shywanglei@qdu.edu.cn
}

\begin{abstract}
A characterization of the equilibrium of information flow networks and the dynamics of network formation are studied under the premise of local information flow. The main result of this paper is that it gives the dynamic formation procedure in the local information flow network. The research shows that core-periphery structure is the most representative equilibrium network in the case of the local information flow without information decay whatever the cost of information is homogeneous or heterogeneous. If the profits and link costs of local information flow networks with information decay are homogeneous empty network and complete network are typical equilibrium networks, which are related to the costs of linking.
\end{abstract}

Keywords local information flow; information decay; dynamic procedure; core-periphery architecture; equilibrium network

\section{Introduction}

The main actors of an information flow network are players. The difference between the information flow network and a regular network is that the equilibrium of information flow

Received February 13, 2015, accepted April 8, 2015

*The corresponding author, E-mail: qiaohan@ucas.ac.cn

The first version of this paper was presented at the $4^{\text {th }}$ International Conference Game Theory and Management (GTM 2010), June 28-30, 2010, St. Petersburg, Russia.

This research was supported by the Russian Foundation for Basic Research (14-01-31141), Saint Petersburg State University (9.38.245.2014), the National Natural Science Foundation of China (71003057, 71171120, 71373262), Specialized Research Fund for the Doctoral Program of Higher Education (20133706110002), Projects of International (Regional) Cooperation and Exchanges of NSFC (71411130215), Graduate Student Education Innovation Plan of Qingdao University (QDY12017, QDY13004) 
network depends on its topological structure and players have the need and competence of acquiring information personally. The dynamic formation procedure of information flow network is one of the most worthy of concern questions ${ }^{[1]}$. In actual practice individuals decide on information acquisition and links with others over time, and it is important to understand these dynamics. Similar to "Two-way" flow network model in common sense ${ }^{[2]}$, an important assumption is that "unilateral formation connection and bilateral information exchange", that is to say, a link is formed once some player pays for it and it allows both players to access the information personally acquired by the other player.

In this paper, we study the characterization of the equilibrium of local information flow networks and the dynamics of network formation. We give the dynamic formation procedure of the local information flow network. The research shows that core-periphery structure is the most representative equilibrium network in the case of the local information flow without information decay whatever the cost of information is homogeneous or heterogeneous. If the profits and link costs of local information flow networks with information decay are homogeneous empty network and complete network are typical equilibrium networks, which are related to the costs of linking.

This work is related to a number of literatures. First, there is a theoretic literature on social networks from a game perspective ${ }^{[3-14]}$. Second, there is an extensive literature from the applying perspective ${ }^{[15-18]}$.

\section{Local Information Flow Networks Without Information Decay}

\subsection{Equilibrium Networks in the Static Case}

Let $N=\{1,2, \cdots, n\}$ with $n \geq 3$ be the set of players and let $i$ and $j$ be typical members of this set. Each player chooses a level of personal information acquisition $x_{i} \in X=[0,+\infty)$ and a set of links with others to access their information, which is represented as a (row) vector:

$$
g_{i}=\left(g_{i 1}, \cdots, g_{i i-1}, g_{i i}, g_{i i+1}, \cdots, g_{i n}\right)
$$

where $g_{i i}=0, \forall i \in N$ and $g_{i j} \in\{0,1\}$, for each $j \in N \backslash\{i\}$. We say that player $i$ has a link with player $j$ if $g_{i j}=1$, and the cost of linking with one other person is $k>0$. Otherwise we have $g_{i j}=0$. Our paper assumes that the cost of linking with one other person is homogeneous, and the link between player $i$ and $j$ allow both players share information. The set of strategies of player $i$ is denoted by $S_{i}=X \times G_{i}$. Define $S=S_{1} \times S_{2} \times \cdots \times S_{n}$ as the set of strategies of all players. A strategy profile $s=(x, g) \in S$ specifies the personal information acquired by each player $x=\left(x_{1}, x_{2}, \cdots, x_{n}\right)$, and the network of relations (connection Matrix) $g=$ $\left(g_{1}, g_{2}, \cdots, g_{n}\right)^{\mathrm{T}}$, where $\mathrm{T}$ specifies a transposition.

The network $g$ is a directed graph, where the arrow from $i$ to $j$ specifies $g_{i j}=1$. Let $G$ be the set of all possible directed graphs on $n$ vertices. Define $N_{i}(g)=\left\{j \in N: g_{i j}=1\right\}$ as the set of players with whom $i$ has formed a link. Let $\eta_{i}(g)=\left|N_{i}(g)\right|$. The closure of $g$ is an undirected network denoted by $\bar{g}=c l(g)$, where $\bar{g}_{i j}=\max \left\{g_{i j}, g_{j i}\right\}$. In words, the closure of a directed network involves replacing every directed edge of by an undirected one. Define $N_{i}(\bar{g})=\left\{j \in N: \bar{g}_{i j}=1\right\}$ as the set of players directly connected to $i$. The undirected link between two players reflects bilateral information exchange between them. 
The payoff to player $i$ under strategy profile $s=(x, g)$ is

$$
\Pi_{i}(s)=f\left(x_{i}+\sum_{j \in N_{i}(\bar{g})} x_{j}\right)-c_{i} x_{i}-\eta_{i}(g) k
$$

where $c_{i}>0$ is the cost of information. We assume the cost of information that personally acquired is heterogeneous. We also assume that $f(y)$ is twice continuously differentiable, increasing, and strictly concave in $y$. To focus on interesting cases we assume

$$
f(0)=0, \quad f^{\prime}(0)>c^{0}=\max _{1 \leq i \leq n}\left\{c_{i}\right\}
$$

and

$$
\lim _{y \rightarrow+\infty} f^{\prime}(y)=m<c_{0}=\min _{1 \leq i \leq n}\left\{c_{i}\right\}
$$

Under these assumptions, there exists a number $\hat{y}_{i}>0$ such that

$$
\hat{y}_{i}=\arg \max _{y \in X}\left[f(y)-c_{i} y\right]
$$

i.e., $\hat{y}_{i}$ solves $f^{\prime}\left(\hat{y}_{i}\right)=c_{i}$.

A Nash equilibrium is a strategy profile $s^{*}=\left(x^{*}, g^{*}\right)$ satisfying

$$
\Pi_{i}\left(s_{i}^{*}, s_{-i}^{*}\right) \geq \Pi_{i}\left(s_{i}, s_{-i}^{*}\right), \quad \forall s_{i} \in S_{i}, \forall i \in N
$$

Example 1 Let $N=\{1,2,3,4,5,6\}$ be the set of players, the cost of linking with one other person be homogeneous $k=\frac{1}{5}$, the cost of information that personally acquired be heterogeneous and denoted by $c_{1}=c_{2}=c_{3}=c_{4}=\frac{1}{2}, c_{5}=c_{6}=\frac{10}{21}$. Suppose that the payoffs are given by $(1)$, where $f(y)=\ln (1+y)$. We can check that $s^{*}=\left(x^{*}, g^{*}\right)$ is a Nash equilibrium, where $x^{*}=\left(x_{1}^{*}, x_{2}^{*}, x_{3}^{*}, x_{4}^{*}, x_{5}^{*}, x_{6}^{*}\right)=\left(\frac{12}{70}, \frac{12}{70}, \frac{12}{70}, \frac{12}{70}, \frac{29}{70}, \frac{29}{70}\right)$, the corresponding connection matrix and the network structure are given below:

$$
g^{*}=\left(\begin{array}{c}
g_{1}^{*} \\
g_{2}^{*} \\
g_{3}^{*} \\
g_{4}^{*} \\
g_{5}^{*} \\
g_{6}^{*}
\end{array}\right)=\left(\begin{array}{cccccc}
0 & 0 & 0 & 0 & 1 & 1 \\
0 & 0 & 0 & 0 & 1 & 1 \\
0 & 0 & 0 & 0 & 1 & 1 \\
0 & 0 & 0 & 0 & 1 & 1 \\
0 & 0 & 0 & 0 & 0 & 0 \\
0 & 0 & 0 & 0 & 0 & 0
\end{array}\right)
$$

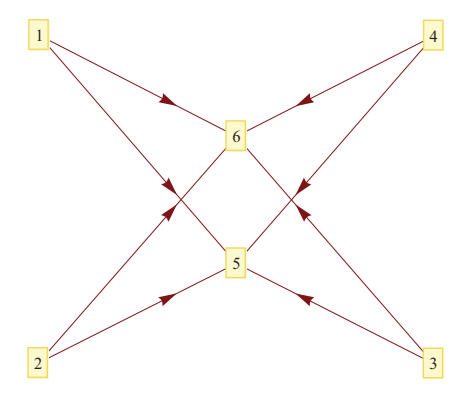

Figure 1 A network 
Notice that equilibrium network in this example has typical "two-core-periphery" architecture. Player 5 and Player 6 become two hubs because they have slightly lower costs of acquiring information, the information that they acquire personally is $\frac{29}{70}$. Players $1,2,3$ and 4 become spokes. Because the cost of linking with one other person is low, so the information that they acquire personally is $\frac{12}{70}\left(x_{1}^{*}=x_{2}^{*}=x_{3}^{*}=x_{4}^{*}=\frac{12}{70}\right)$ and aggregate information is 1 through linking with two hubs, in fact $\hat{y}_{1}=\hat{y}_{2}=\hat{y}_{3}=\hat{y}_{4}=1$ at this moment, the aggregate information which Player 5 and Player 6 own is $\frac{11}{10}=\hat{y}_{5}=\hat{y}_{6}$. Under the equilibrium network, the payoff of spokes is

$$
\Pi_{i}\left(s^{*}\right)=\ln \left(1+\hat{y}_{i}\right)-c_{i} x_{i}^{*}-2 k=\ln (1+1)-\frac{1}{2} \times \frac{6}{35}-2 \times \frac{1}{5}=0.2074, \quad i=1,2,3,4
$$

However, when spokes acquire the optimal aggregate information 1 personally and do not link with hubs, the payoff is

$$
\Pi_{i}(s)=\ln \left(1+\hat{y}_{i}\right)-c_{i}=\ln (1+1)-\frac{1}{2}=0.193, \quad i=1,2,3,4
$$

Similarly, we can verify $\Pi_{i}(s)<\Pi_{i}\left(s^{*}\right), \forall s \in S_{i}, \forall i \in N$. So the spokes form links with two hubs while do not form links between them and two hubs do not form links between them is the equilibrium strategy for the players.

In this example, there is no link between two hubs because of $k>c_{5} \hat{y}_{6}=c_{6} \hat{y}_{5}\left(0.2>\frac{10}{21} \times\right.$ $\left.\frac{29}{70}=0.1973\right)$. Spokes choose to form links with hubs because of $k<c_{i} \hat{y}_{5}=c_{i} \hat{y}_{6}(i=1,2,3,4)$, i.e. $0.2<\frac{1}{2} \times \frac{29}{70}=0.2071$.

As we see in Example 1, the cost heterogeneity of information acquisition is based on two levels. The primary cause lies in the high complexity of the algorithm of general equilibrium network. In fact, slight cost difference can help us distinguish which players attain information actively and which players become spokes.

To simplify symbolic system, we mark $\hat{y}_{1}$ and $\hat{y}$ as the optimal value of information, which are obtained by players who have information cost advantage and have not advantage. We use $\tilde{c}=c-\varepsilon<c$ to express the slight advantage of attaining information cost, where $\varepsilon>0$ is a small number.

In the network $g$ with core-periphery architecture, we assume that $N_{c}(g)$ be the set of hubs, where $\left|N_{c}(g)\right|=m$, then $N \backslash N_{c}(g)$ be the set of spokes, and we have $\left|N \backslash N_{c}(g)\right|=m q$, where $q \in N_{+}$, namely $n=(q+1) m$. The homogeneous cost of linking with one other person is $k$ which satisfies $f(\hat{y})-c \hat{y}<f\left(m \hat{y}_{1}\right)-m k$.

Lemma 1 In the equilibrium networks with core-periphery architecture, if for each $l \in N$, we have $x_{l}>0$, then $x_{i}+y_{i}=\hat{y}_{1}$, for each $i \in N_{c}(g)$. Moreover, $x_{p}+y_{p}=\hat{y}$, for each $p \in N \backslash N_{c}(g)$.

The proof of this lemma is similar to Lemma 1 in [1].

Definition 1 The local information flow network is called core-empty-periphery, if for any pair of players $i, i^{\prime} \in N_{c}(g)$, we have $\bar{g}_{i i^{\prime}}=0$, and for each player $p \in N \backslash N_{c}(g)$, we have $g_{p 1}=g_{p 2}=\cdots=g_{p m}=1$.

Definition 2 The local information flow network is called core-completely-periphery, if for any pair of players $i, i^{\prime} \in N_{c}(g)$, we have $\bar{g}_{i i^{\prime}}=1$, and for each player $p \in N \backslash N_{c}(g)$, we have $g_{p 1}=g_{p 2}=\cdots=g_{p m}=1$. 
Theorem 1 The local information flow network is a equilibrium network with "core-emptyperiphery" architecture, if

1) the personal information acquired by each player $i$ is

$$
x_{i}=\hat{y}\left(\frac{m q}{m^{2} q-1}\right)-\left(\frac{1}{m^{2} q-1}\right) \hat{y}_{1}, \quad \forall i \in N_{c}(g)
$$

2) the personal information acquired by each player $p$ is

$$
x_{p}=\frac{m \hat{y}_{1}-\hat{y}}{m^{2} q-1}, \quad \forall p \in N \backslash N_{c}(g)
$$

3) $c x_{p}<k<c x_{i}, k>\widetilde{c} x_{i}, \frac{c}{k}>\frac{m}{\hat{y}-x_{p}}$, where $i \in N_{c}(g), p \in N \backslash N_{c}(g)$.

In fact, the first inequality in term 3 ) assures that spokes are not being connected and spokes are favorable to link with hubs; the second inequality assures that hubs are not being connected; the third inequality assures that spokes link with hubs if they acquire partial information actively. Meeting with information level of terms 1) and 2) can guarantee hubs owning the aggregate information $\hat{y}_{1}$ in the network, while the aggregate information owned by spokes is $\hat{y}$. It is shown in Example 1.

Theorem 2 The local information flow network is a equilibrium network with "corecompletely-periphery" architecture, if

1) The personal information acquired by each player $i$ is

$$
\frac{q}{m q-1} \hat{y}-\frac{1}{m^{2} q-m} \hat{y}_{1}, \quad \forall i \in N_{c}(g)
$$

2) The personal information acquired by each player $p$ is

$$
x_{p}=\frac{\hat{y}_{1}-\hat{y}}{m q-1}, \quad \forall p \in N \backslash N_{c}(g)
$$

3) $c x_{p}<k<\widetilde{c} x_{i}<c x_{i}, \frac{c}{k}>\frac{m}{\hat{y}-x_{p}}$, where $i \in N_{c}(g), p \in N \backslash N_{c}(g)$.

Similar to Theorem 1, the first inequality in term 3) assures that spokes do not form links between them while hubs are favorable to form links with each other and spokes are favorable to form links with hubs; the second inequality assures that spokes link with hubs if they acquire partial information actively. Meeting with information level of terms 1) and 2) can guarantee hubs own the aggregate information $\hat{y}_{1}$ in the network, while the aggregate information owned by spokes is $\hat{y}$.

We can see from the above situation, after fixing the amount of hubs, the information acquisition of spokes is degressive. So if $q \longrightarrow+\infty$, then $x_{p} \longrightarrow 0$. Relatively, the information acquisition of hubs is increasing.

The result also reflects contents of "The law of the few", that is a lot of information will be grasped in a few hubs, while most of other players, that is, the spokes will choose to link with hubs to get information, but themselves will choose little "personal information acquisition", or even entirely depends on the connection to get information, making their "personal information acquisition" to zero.

Example 2 Let $N=\{1,2,3,4,5,6,7,8,9\}$ be the set of players, the cost of linking with one other person be homogeneous, we denote by $k=0.15$, the cost of information that personally acquired be heterogeneous and is denoted by $c_{1}=c_{2}=c_{3}=c_{4}=c_{5}=c_{6}=\frac{1}{2}, c_{7}=c_{8}=c_{9}=$ 
$\frac{10}{21}$. Suppose that payoffs are given by $(1)$, where $f(y)=\ln (1+y)$. The initial matrix is a zero matrix, and the initial information vector is $\left(0,0,0,0,0,0, \frac{11}{30}, \frac{11}{30}, \frac{11}{30}\right)$.

We can check that $s^{*}=\left(x^{*}, g^{*}\right)$ is a Nash equilibrium, where

$$
x^{*}=\left(0,0,0,0,0,0, \frac{11}{30}, \frac{11}{30}, \frac{11}{30}\right), \quad g^{*}=\left(\begin{array}{c}
g_{1}^{*} \\
g_{2}^{*} \\
g_{3}^{*} \\
g_{4}^{*} \\
g_{5}^{*} \\
g_{6}^{*} \\
g_{7}^{*} \\
g_{8}^{*} \\
g_{9}^{*}
\end{array}\right)=\left(\begin{array}{ccccccccc}
0 & 0 & 0 & 0 & 0 & 0 & 0 & 1 & 1 \\
0 & 0 & 0 & 0 & 0 & 0 & 0 & 1 & 1 \\
0 & 0 & 0 & 0 & 0 & 0 & 0 & 1 & 1 \\
0 & 0 & 0 & 0 & 0 & 0 & 0 & 1 & 1 \\
0 & 0 & 0 & 0 & 0 & 0 & 0 & 1 & 1 \\
0 & 0 & 0 & 0 & 0 & 0 & 0 & 1 & 1 \\
0 & 0 & 0 & 0 & 0 & 0 & 0 & 1 & 1 \\
0 & 0 & 0 & 0 & 0 & 0 & 0 & 0 & 1 \\
0 & 0 & 0 & 0 & 0 & 0 & 0 & 0 & 0
\end{array}\right)
$$

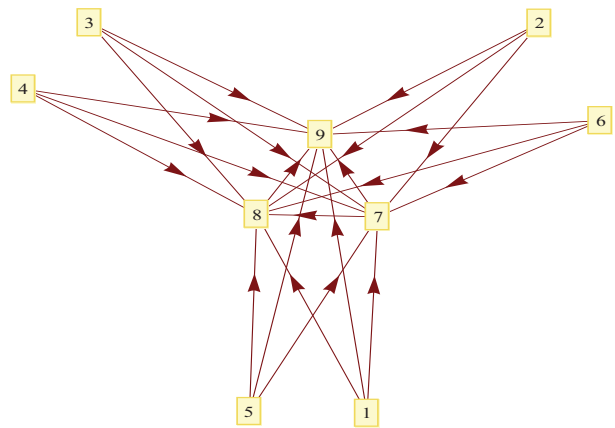

Figure 2 A three-core-completely-periphery equilibrium network

\subsection{A Dynamic Formation Procedure and the Algorithm}

The algorithm of finding equilibrium networks in this paper is based on a dynamic procedure used in $[12,19,20]$.

Given the set of players, at each stage, agents who are chosen at random play their best response, adjust their links in response to the network structure and the personal information acquired by oneself in previous period and then compose the network structure and the personal information (it is called the player's non-coordinated behavior).

At stage $t$, first select a subset $R$ of the set of players $N$ at random. For every selected player $i \in R$, we calculate his optimal personal information and the best link set by comparing all of his possible links, namely the player's best response strategy. Each player who is selected at random chooses a pure strategy best response to the strategy of all other agents in the previous period and for the player who is not selected he maintains the strategy chosen in the previous period. Compose all strategies, and then dynamic procedure comes to stage $t+1$.

Based on above dynamic procedure, the advantage of the algorithm is that the optimization question has only one variable. The network reaches a stable state when no one chooses to 
change his links and personal information during dynamic procedure, and then we can get equilibrium strategy profile $s^{*}=\left(x^{*}, g^{*}\right)$ and relative information equilibrium network.

Given an initial stage $t_{0}$, an initial information vector $x^{t_{0}}=\left(x_{1}^{t_{0}}, x_{2}^{t_{0}}, \cdots, x_{n}^{t_{0}}\right)$, initial link matrix $\left[\alpha_{i j}^{t_{0}}\right] \in\{0,1\}^{N \times N}$, where $\alpha_{i i}^{t_{0}}=0, i \in N$ and $\alpha_{i j}^{t_{0}} \in\{0,1\}, \forall j \in N \backslash\{i\}$. Homogeneous link costs $k \geq 0$ and heterogeneity $\operatorname{costs} c_{i} \geq 0$ are given.

At stage $t$, let information vector be $x^{t}=\left(x_{1}^{t}, x_{2}^{t}, \cdots, x_{n}^{t}\right)$, link matrix $\left[\alpha_{i j}^{t}\right]=\left(\alpha_{1}^{t}, \alpha_{2}^{t}, \cdots\right.$, $\left.\alpha_{n}^{t}\right)^{\mathrm{T}} \in\{0,1\}^{N \times N}$, where $\alpha_{i}^{t}$ is the row $i$ of the link matrix $\left[\alpha_{i j}^{t}\right]$.

Select randomly the set of players $R=\left(i_{1}, i_{2}, \cdots, i_{r}\right) \subseteq N$. For every $i_{k} \in R$, the row $i_{k}$ of link matrix has $2^{n-1}$ possibilities if we fix the rest $n-1$ rows. For every possibility, first calculate $\bar{\alpha}_{i_{k} j}^{t}=\max \left\{\alpha_{i_{k} j}^{t}, \alpha_{j i_{k}}^{t}\right\} \in\{0,1\}$, it is easy to see $\bar{\alpha}_{i_{k} i_{k}}^{t}=0$.

Consider that

$$
\max \theta_{i_{k}}^{t}=f\left(x_{i_{k}}^{t}+\sum_{j \in N} \bar{\alpha}_{i_{k} j}^{t} x_{j}^{t}\right)-c_{i_{k}} x_{i_{k}}^{t}-\sum_{j \in N} \alpha_{i_{k} j}^{t} k
$$

subject to $x_{i_{k}}^{t} \geq 0$.

Notice that $\sum_{j \in N} \bar{\alpha}_{i_{k} j}^{t} x_{j}^{t}$ and $\sum_{j \in N} \alpha_{i_{k} j}^{t} k$ in objective function are constant for given stage $t$ and all probably value of line $i_{k}$ in link matrix $\left[\alpha_{i j}^{t}\right]$. So the variable in (3) is $x_{i_{k}}^{t}$.

Suppose that the optimal value is reached at $x_{i_{k}}^{t}$. For all the possibilities in row $i_{k}$ we calculate relative optimal value $x_{i_{k}}^{t}$ and $\theta_{i_{k}}^{t}$. At last by comparing above $2^{n-1}$ optimal value $\theta_{i_{k}}^{t}$, we can find $\max \theta_{i_{k}}^{t}$, and then output relative optimal value $\widetilde{x}_{i_{k}}^{t}$ and $\widetilde{\alpha}_{i_{k}}^{t}$.

Let

$$
\left[\alpha_{i j}^{t+1}\right]=\left(\alpha_{1}^{t}, \cdots, \widetilde{\alpha}_{i_{1}}^{t}, \cdots, \widetilde{\alpha}_{i_{r}}^{t}, \cdots, \alpha_{n}^{t}\right)^{\mathrm{T}}
$$

be link matrix at stage $t+1$, and information vector

$$
x^{t+1}=\left(x_{1}^{t+1}, \cdots, x_{n}^{t+1}\right)=\left(x_{1}^{t}, \cdots, \widetilde{x}_{i_{1}}^{t}, \cdots, \widetilde{x}_{i_{r}}^{t}, \cdots, x_{n}^{t}\right)^{\mathrm{T}}
$$

The dynamic procedure comes into stage $t+1$. Select the set of players at random again and the network formation procedure is repeated.

The dynamic procedure is end until no one chooses to change his links and personal information. At last we get the information flow equilibrium network and equilibrium strategy profile will comprise the link matrix of the equilibrium network and the optimal personal acquired information vector. It should be noticed that to guarantee the valid of the algorithm we must suppose that the dynamic procedure is not a circulation.

Example 3 Given the set of players $N=\{1,2,3,4,5,6\}$, homogeneous link costs $k=$ 0.04 and personal information heterogeneity costs $c_{1}=c_{2}=c_{3}=c_{4}=\frac{1}{10}, c_{5}=c_{6}=\frac{1}{11}$. Payoff function is given by (1), where $f(y)=\ln (1+y)$. The initial link matrix and the initial information vector are 0 .

It is different to reach final stage for each implementation of the dynamic process program and convergence to the equilibrium state because the non-coordinated behavior of the players caused by the randomness of process of forming network. Therefore there is not practical significance for number of stages to achieve an equilibrium state.

We only had one interception of several typical stages in some run results in Figure 3, and let $K$ be the number of stages needed to achieve an equilibrium state.

The network structure and dynamics of information vectors in Example 3: 
1) At the initial stage we select the set of players $R=\{2,3,4,5,6\}$ at random, because of the initial matrix is zero, they all choose to acquire their optimal aggregate information and do not form any link. And then it will come into an empty network by the first round of iteration. But the information level vector is $x^{1}=\left(x_{1}^{1}, \cdots, x_{6}^{1}\right)=(0,9,9,9,10,10)$.

2 ) If the selected set of players is $R=\{3,5,6\}$ at stage 1 , we can infer that it is the best response for Player 3 to form links with Players 2, 4, 5 and 6 and he do not acquire information personally, the payoff $\Pi_{3}()=.\ln [1+(9+9+10+10)]-4 \times 0.04 \approx 3.5036$ is larger than the payoff in any other situation. Because Players 5 and 6 have the same acquired advantage, it is the best response for Players 5 and 6 to form links with 2, 3, 4, 6 and he do not acquire information. The dynamic procedure comes into stage 2 .

3) When the dynamic procedure comes into stage $t_{1}$, the current network has two-coreperiphery architecture. The information level vector of players is $x^{t_{1}}=(0,9,0,0,0,0)$. Select set of the players $R=\{1,4,5,6\}$ randomly. Although here the two-core-periphery architecture is the same as the eventual equilibrium structure formally (Players 2 and 4 are temporal core), obviously, for Players 5 and 6 their aggregate information do not reach their optimal value $\hat{y}_{5}=\hat{y}_{6}=10$, so here the network is not an equilibrium network and not stable. For Players 5 and 6 they will delete their links with Player 4 and maintain their link with Player 2 and acquire information 1 by themselves, the payoff $\Pi_{i}(\cdot)=\ln [1+(9+1)]-\frac{1}{11} \times 1-0.04 \approx 2.2669, i=5,6$ is lager than in any other situation, so it is the best response for them on this stage. Because Player 4 dose not acquire any information personally, Players 1, 5 and 6 will delete their links with 4 and Players 1 and 4 can acquire their optimal aggregate information 9 by maintaining their links with 2 .

4) When the dynamic procedure comes into stage $t_{2}$, the current network has three-corecompletely-periphery architecture. The information level of players is $x^{t_{2}}=(0,3,1,0,5,1)$. Selected set of the players $R=N$. So here for Players 1 and 4 it is their best response to maintain their links with Players 2, 3, 5 and 6 . Players 2 and 3 will maintain their links and reduce their information 1 by themselves and for Players 5 and 6 they maintain their strategies from the previous period. Notice that here the network is four-core-completely-periphery architecture, it is not stable.

5) When the dynamic procedure comes into stage $K-1$, the information level of players is $x^{K-1}=(0,0,0,0,8,2)$. Selected set of the players $R=\{1\}$ randomly. For Player 1 his best response is to delete his link with Player 3 and maintain his link with 5 and 6 while he acquires no information. The "two-core-periphery" equilibrium network is formed. The hub is composed of two players with information cost advantage acquired actively. The dynamic procedure is over.

Core-periphery structure is the most representative equilibrium network in the case of the local information flow without information loss. In fact, under the premise of players have two levels of information each person who has a comparative advantage of the cost of acquiring information would become the hub possibly. The statistical results show that it is the easiest to form "single-core-periphery" structure; but the "multi-core-periphery" structure which contains all players who has the same advantage on the cost as the hubs (maximum) has the smallest probability; those which between these two kinds become harder and harder with the increasing 
of the players' number in the core.

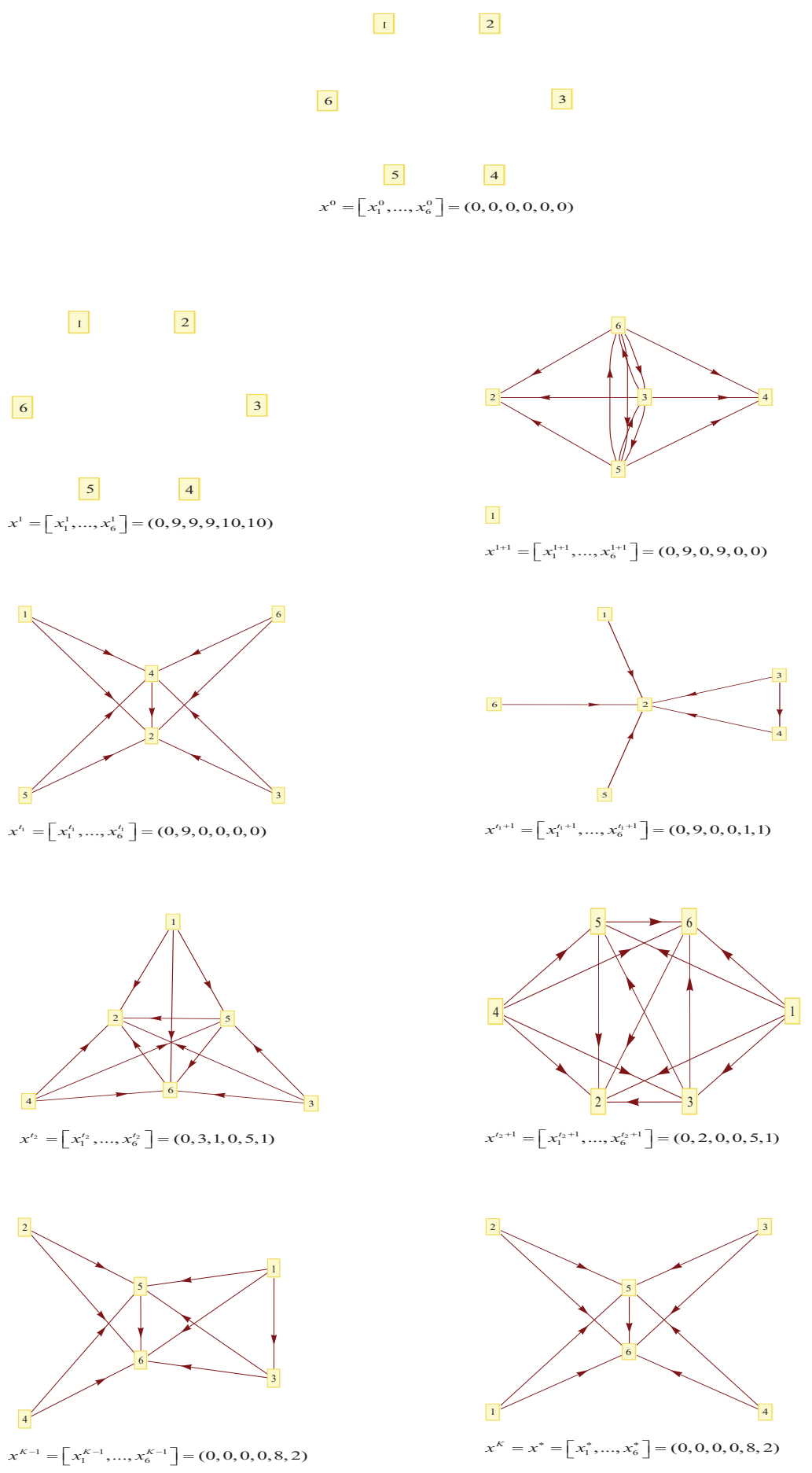

Figure 3 Stage examples of the dynamic process 
We believe that there are several reasons why the dynamics are important. One of the reasons is that a dynamic model allows us to study the process by which individual agents learn about the network and adjust their links in response to their learning. And the dynamics may help select among different equilibria of the static game.

There is very important prospect to study the characterization of the architecture of information flow equilibrium networks and the dynamics of network formation under the premise of local information flow and without the presence of decay. In addition, consider that some of the players may coordinate and prepare to maximize the common interests of members by some local cooperation; the model and algorithm in this essay can be transformed to an incomplete information cooperative game model. This article assumes that the players have the need and competence of acquiring information personally and getting information from others in the given network structure, in fact, this can also be diversified.

\section{Local Information Flow Networks with Information Decay}

Lemma 2 In the local information flow equilibrium network $g$ with information decay, we have $x_{i}+y_{i} \geq \hat{y}$, for all $i \in N$, and if $x_{i}>0$, then $x_{i}+y_{i}=\hat{y}$. Here $y_{i}=\sum_{j \in N_{i}(\bar{g})} \delta x_{j}$, that is to say $y_{i}$ represents the information that Player $i$ acquires from his neighbors.

Proof Suppose not, then there must exist some Player $i$ such that $x_{i}+y_{i}<\hat{y}$ in equilibrium network $g$. Under the assumptions that $f(y)$ is twice continuously differentiable, increasing, and strictly concave in $y$, we know $f^{\prime}\left(x_{i}+y_{i}\right)>c$, so Player $i$ can strictly increase his payoffs by increasing personal information acquisition, a contradiction with equilibrium. So we know $x_{i}+y_{i} \leq \hat{y}, \forall i \in N$. Next suppose that $x_{i}>0$ and $x_{i}+y_{i}>\hat{y}$. Under our assumptions on $f(\cdot)$ and $c$, if $x_{i}+y_{i}>\hat{y}$ then $f^{\prime}\left(x_{i}+y_{i}\right)<c$, and then Player $i$ can strictly increase his payoffs by lowering personal information acquisition, which contradicts equilibrium. Therefore, if $x_{i}>0$, then $x_{i}+y_{i}=\hat{y}$.

Theorem 3 In the local information flow equilibrium network with information decay, if $k>c \delta \hat{y}$, the empty network is unique equilibrium. Every player acquires information $\hat{y}$ personally and no one forms links.

Proof 1) Suppose that the strategy profile $s=(x, g)$ corresponds to an empty network. If Player $i$ forms $m_{1}$ links with other $m_{1}$ players and personally acquires information $x_{i}^{\prime}$, here the strategy profile is $s \mid s_{i}^{\prime}$. Lemma 2 implies that the aggregate information of Player $i$ should be $\hat{y}$, and then his payoff is

$$
\Pi_{i}\left(s \mid s_{i}^{\prime}\right)=f(\hat{y})-c\left(\hat{y}-\delta m_{1} \hat{y}\right)-m_{1} k
$$

however, in the empty networks, his payoff is

$$
\Pi_{i}(s)=f(\hat{y})-c \hat{y}
$$

Since $k>c \delta \hat{y}$, we have

$$
\Pi_{i}(s)>\Pi_{i}\left(s \mid s_{i}^{\prime}\right)
$$

2) Suppose Player $i$ forms $m_{2}$ links with other $m_{2}$ players initiatively based on the empty network, and $x_{i}^{\prime \prime}=0$, the strategy profile is $s \mid s_{i}^{\prime \prime}$, payoff is

$$
\Pi_{i}\left(s \mid s_{i}^{\prime \prime}\right)=f\left(\delta m_{2} \hat{y}\right)-m_{2} k
$$


Here $\delta m_{2} \hat{y} \geq \hat{y}$, then $\delta m_{2} \geq 1$. Therefore

$$
\Pi_{i}\left(s \mid s_{i}^{\prime \prime}\right)=f\left(\delta m_{2} \hat{y}\right)-m_{2} k<f\left(\delta m_{2} \hat{y}\right)-c m_{2} \delta \hat{y} \leq f(\hat{y})-c \hat{y}=\Pi_{i}(s)
$$

Due to $k>c \delta \hat{y}$, the first inequality is strict obviously, since $f(y)$ is twice continuously differentiable, increasing, and strictly concave in $y$, the second is hold. Then the empty network is an equilibrium network.

Finally, we will show that the empty network is the unique equilibrium. As we know, every player's personal information acquisition is no more than $\hat{y}$, if some Player $i$ wants to form a link with other player (say $j$ ), Player $i$ can obtain $\delta x_{j}$ from $j$, and $c \delta x_{j}>k$, this would contradict with $k>c \delta \hat{y}$ (because $\hat{y}>x_{j}$ ).

Lemma 3 If complete network is the local information flow equilibrium network with information decay, the personal information acquisition of each player equals $x=\frac{\hat{y}}{1+(n-1) \delta}$.

Proof For every player he acquires information personal $x_{i} \geq 0$. If there are $m(1 \leq m<n)$ players who personally acquires information 0 , we let $x_{1}=x_{2}=\cdots=x_{m}=0$, according to Lemma 2, we conclude following inequalities:

$$
\left\{\begin{array}{l}
\sum_{i \in N \backslash\{1,2, \cdots, m\}} \delta x_{i} \geq \hat{y} \\
x_{j}+\sum_{i \in N \backslash\{j\}} \delta x_{i}=\hat{y}, \quad j=m+1, m+2, \cdots, n
\end{array}\right.
$$

We can obtain $x_{m+1}=\cdots=x_{n}=\frac{\hat{y}}{1+(n-m-1) \delta}$ from the second inequality, hence, $\delta \geq 1$ a contradiction. Hence, in complete network, we have $x_{i}>0, \forall i \in N$. By Lemma 2, we have

$$
x_{i}+\sum_{j \in N \backslash\{i\}} \delta x_{j}=\hat{y}, \quad i=1,2, \cdots, n
$$

therefore

$$
x_{1}=x_{2}=\cdots=x_{n}=x=\frac{\hat{y}}{1+(n-1) \delta}
$$

Theorem 4 In the local information flow equilibrium network with information decay, if $k<c \delta x=\frac{c \delta \hat{y}}{1+(n-1) \delta}$, complete network is the unique equilibrium.

Proof Firstly prove that if the personal information acquisition of each player equals $x=$ $\frac{\hat{y}}{1+(n-1) \delta}$ in the complete network, the complete network is an equilibrium structure. Let $s^{*}=\left(s_{1}^{*}, s_{2}^{*}, \cdots, s_{n}^{*}\right)$ be the strategy profile in complete network, where $s_{i}^{*}=\left(g_{i}^{*}, x\right)$. If Player $i$ has $m \quad(0 \leq m \leq n-1)$ links which he formed with others, since every player can obtain information from other $n-1$ players, they would not form links with others.

Secondly, we show that it is not the best response for Player $i$ of deleting links or changing his personal information level.

Now consider the case $m=0$, Player $i$ can change their personal information acquisition to be $x^{\prime}$, we can write strategy profile $s^{*} \mid s_{i}^{\prime}$, hence

$$
\Pi_{i}\left(s^{*} \mid s_{i}^{\prime}\right)=f\left(\hat{y}-x+x^{\prime}\right)-c x^{\prime}<f(\hat{y})-c x=\Pi_{i}\left(s^{*}\right)
$$

so changing strategies is not the best response for Player $i$.

Consider the case $m=1$, if Player $i$ deletes links and acquires information $(1+\delta) x$ personally, hence

$$
\Pi_{i}\left(s^{*} \mid s_{i}^{\prime}\right)=f(\hat{y})-c(1+\delta) x
$$


Since $k<c \delta x$,

$$
\Pi_{i}\left(s^{*} \mid s_{i}^{\prime}\right)=f(\hat{y})-c(1+\delta) x<f(\hat{y})-c x-k=\Pi_{i}\left(s^{*}\right)
$$

so changing strategies is not the best response for Player $i$.

Similarly, we can show that the best response for Player $i$ is to maintain current strategy and completeness of network when $m=2,3, \cdots, n-1$ and $k<c \delta x$.

In the following we show that if $k<c \delta x=\frac{c \delta \hat{y}}{1+(n-1) \delta}$, each player acquires information $x=\frac{\hat{y}}{1+(n-1) \delta}$ personally, complete network is the unique equilibrium.

Lemma 3 implies that if a complete network is the equilibrium structure, the personal information acquisition of each player equals $x=\frac{\hat{y}}{1+(n-1) \delta}$.

Two steps: The first step is to prove the connected but not completely network is not equilibrium, the second step is to prove not connected network is not the equilibrium.

Step 1:

Suppose the connected but not completely network is an equilibrium. Noticed that the equilibrium network must be essential ${ }^{[2]}$.

We delete any link in complete network, $g_{i j}=0$ or $g_{j i}=0$, let $g_{1}$ be the network. Suppose $g_{1}$ is equilibrium. By Lemma 2, every player's aggregate information should be more than or equal to $\hat{y}$. Since $\bar{g}_{i j}=0$, we have $k>c \delta x_{i}$ and $k>c \delta x_{j}$. Players who are in $N \backslash\{i, j\}$ have links with each other, let $N_{1}=\{i, j\}, N_{2}=N \backslash\{i, j\}, I(s)=\left\{p \mid x_{p}>0, p \in N\right\}$. For $q \in N_{2} \cap I(s)$, we have

$$
x_{q}+\sum_{p \in\left(N_{2} \cap I(s)\right) \backslash\{q\}} \delta x_{p}+\sum_{t \in N_{1}} \delta x_{t}=\hat{y}
$$

If $x_{i}=x_{j}=0$, and $i, j \in N_{1}$, so that

$$
\begin{aligned}
& x_{i}+\sum_{p \in N_{2} \cap I(s)} \delta x_{p}=0+\sum_{p \in N_{2} \cap I(s)} \delta x_{p}<\hat{y} \\
& x_{j}+\sum_{p \in N_{2} \cap I(s)} \delta x_{p}=0+\sum_{p \in N_{2} \cap I(s)} \delta x_{p}<\hat{y}
\end{aligned}
$$

a contradiction with equilibrium.

If $x_{i}=0, x_{j}>0$, and $i \in N_{1}$, hence

$$
x_{i}+\sum_{p \in N_{2} \cap I(s)} \delta x_{p}=0+\sum_{p \in N_{2} \cap I(s)} \delta x_{p}<\hat{y}
$$

then Player $i$ can strictly increase his payoffs by increasing personal information acquisition. It contradicts with equilibrium.

The case of $x_{j}=0, x_{i}>0$ is similar to the above.

Hence, we can conclude that personal information acquisition of $i$ and $j$ more than 0 . Otherwise, for $l \in N_{2} \backslash I(s)$, we have

$$
\begin{aligned}
x_{l}+\sum_{p \in N_{2} \cap I(s)} \delta x_{p}+\sum_{t \in N_{1}} \delta x_{t} & =0+\sum_{p \in N_{2} \cap I(s)} \delta x_{p}+\sum_{t \in N_{1}} \delta x_{t} \\
& =\sum_{p \in\left(N_{2} \cap I(s)\right) \backslash\{q\}} \delta x_{p}+\delta x_{q}+\sum_{t \in N_{1}} \delta x_{t} \\
& <x_{q}+\sum_{p \in\left(N_{2} \cap I(s)\right) \backslash\{q\}} \delta x_{p}+\sum_{t \in N_{1}} \delta x_{t}=\hat{y}
\end{aligned}
$$


That is to say, the aggregate information of $l$ is less than $\hat{y}$, contradiction. Hence, for any $i \in N$, every player's personal information acquisition is $x_{i}>0$. According to Lemma 2, the following inequalities are held

$$
\left\{\begin{array}{l}
x_{t}+\sum_{p \in N_{2}} \delta x_{p}=\hat{y}, \quad \forall t \in N_{1} \\
x_{q}+\sum_{p \in N_{2} \backslash\{q\}} \delta x_{p}+\sum_{t \in N_{1}} \delta x_{t}=\hat{y}, \quad \forall q \in N_{2}
\end{array}\right.
$$

So the personal information acquisition in $N_{1}$ equals to $x_{i}=x_{j}=x^{\prime}$, and the personal information acquisition in $N_{2}$ equals to $x_{q}=x^{\prime \prime}, \forall q \in N_{2}$, so that

$$
\left\{\begin{aligned}
x^{\prime} & =\frac{(1-\delta) \hat{y}}{1+\delta-2 n \delta^{2}+4 \delta^{2}} \\
x^{\prime \prime} & =\frac{(1-2 \delta) \hat{y}}{1+\delta-2 n \delta^{2}+4 \delta^{2}}
\end{aligned}\right.
$$

For any $n \geq 3$, any network is not equilibrium network.

Similarly, we can show that except complete network, connected network is not the equilibrium network.

Step 2:

Firstly, suppose there is an isolated player in equilibrium network at least, the set of these players is $N^{0}$. The personal information acquisition is $\hat{y}$ in $N^{0}$. Due to $k<c \delta x<c \delta \hat{y}$, there must exist some player in $N \backslash N^{0}$ who will strictly increase his payoffs by lowering his personal information acquisition and switching to link with players in $N^{0}$, contradiction with equilibrium.

Secondly, suppose $C_{1}(\bar{g})$ and $C_{2}(\bar{g})$ are two parts of the network $\bar{g}$, and every part has more than one player.

From Step 1, we know that the two parts are complete.

Suppose $\left|C_{1}(\bar{g})\right|=n_{1}>1,\left|C_{2}(\bar{g})\right|=n_{2}>1$, and assume $n_{1} \leq n_{2}$. Lemma 2 and Lemma 3 claimed that the information is equal in $C_{1}(\bar{g})$ and $C_{2}(\bar{g})$, respectively:

$$
x_{n_{1}}=\frac{\hat{y}}{1+\left(n_{1}-1\right) \delta}, \quad x_{n_{2}}=\frac{\hat{y}}{1+\left(n_{2}-1\right) \delta}
$$

Then $x_{n_{1}} \geq x_{n_{2}}>x$. We can concluded that $k<c \delta x<c \delta x_{n_{2}} \leq c \delta x_{n_{1}}$, the players in $C_{2}(\bar{g})$ will link with the players in $C_{1}(\bar{g})$ initiatively. A contradiction completes the proof.

Similarly, we can show that non-connected network which contains multiple parts is not the equilibrium.

Example 4 Let $N=\{1,2,3,4,5,6\}$ be the set of players, the homogeneous cost of linking with one other person is denoted by $k$, the homogeneous cost of information that players acquire is denoted by $c$, the index of local information decay is denoted by $\delta$ and $0<\delta<1$, where payoffs are given by $(1), f(y)=\ln (1+y)$.

As shown in Figure $4, \delta$ is $x$-coordinate, $k$ is $y$-coordinate, where $n=6, c=\frac{1}{3}, \hat{y}=2$, the curve equation is $k=\frac{2 \delta}{3+15 \delta}$, and the linear equation is $k=\frac{2}{3} \delta$. If $k<\frac{2 \delta}{3+15 \delta}$, complete network (Figure 5) is the unique equilibrium, Region C; if $k>\frac{2}{3} \delta$, empty network is the unique equilibrium, Region A; if $\frac{2 \delta}{3+15 \delta}<k<\frac{2}{3} \delta$, there exists no equilibrium, Region B. 


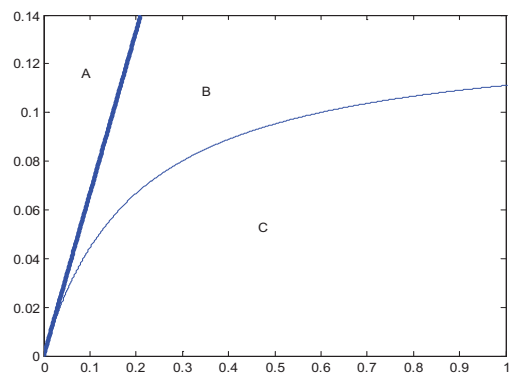

Figure 4 A distribution of equilibrium networks

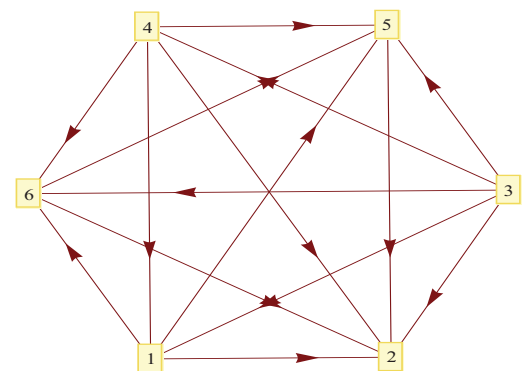

Figure 5 A complete network

But if $c \delta x<k<c \delta \hat{y}, x=\frac{\hat{y}}{1+(n-1) \delta}$, what is the equilibrium structure in the local information flow network with information decay? Many examples show that no equilibrium structure exists in that case. Until now it is still an interesting open problem.

\section{References}

[1] Galeotti A, Goyal S. The law of the few. American Economic Review, 2010, 100(4): 1468-1492.

[2] Bala V, Goyal S. A noncooperative model of network formation. Econometrica, 2000, 68: 1181-1229.

[3] Bala V, Goyal S. A strategic analysis of network reliability. Review of Economic Design, 2000, 5: 205-228.

[4] Barabasi A. Linked. Perseus Books Group: New York, 2002.

[5] Bloch F, Jackson M O. The formation of networks with transfers among players. Journal of Economic Theory, 2007, 133: 83-110.

[6] Galeotti A, Goyal S, Kamphorst J. Network formation with heterogenous players. Games and Economic Behavior, 2006, 54: 353-372.

[7] Galeotti A. One-way flow networks: The role of heterogeneity. Economic Theory, 2006, 29(1): 163-179.

[8] Goyal S, Vega-Redondo F. Network formation and social coordination. Games and Economic Behavior, 2005, 50(2): 178-207.

[9] Goyal S. Connections: An introduction to the economics of networks. Princeton, Princeton University Press, 2007.

[10] Goyal S. Learning in networks. Benhabib J, Bisin A, Jackson M O (eds). Handbook of Social Economics, 2011, 1: 679-727.

[11] Haller H, Sarangi S. Nash networks with heterogeneous links. Mathematical Social Sciences, 2005, 50(2): $181-201$.

[12] Hojman D, Szeidl A. Core and periphery in networks. Journal of Economic Theory, 2008, 139: 295-309.

[13] Jackson M O, Wolinsky A. A strategic model of social and economic networks. Journal of Economic Theory, 1996, 71(1): 44-74.

[14] Jackson M O. Social and economic networks. Princeton, Princeton University Press, 2008.

[15] Che X, Bu H, Liu J J. A theoretical analysis of financial agglomeration in China based on information asymmetry. Journal of Systems Science and Information, 2014, 2(2): 111-129.

[16] Yang Y, Lu X, Qiao H. A robust factor analysis model for dichotomous data. Journal of Systems Science and Information, 2014, 2(5): 437-450.

[17] Xie H B, Bian J Z, Wang M X, Qiao H. Is technical analysis informative in UK stock market? Evidence from decomposition-based vector autoregressive (DVAR) model. Journal of Systems Science and Complexity, 2014, 27(1): 144-156.

[18] Zhao Y X, Hu Z H, Qiao H, Wang S Y, Uria L C. Characterizations of semi-prequasi-invesity. Journal of Systems Science and Complexity, 2014, 27(5): 1008-1026.

[19] Gao H W, Yang H J, Wang G X, et al. The existence theorem of absolute equilibrium about games on connected graph with state payoff vector. Science China Math, 2010, 53(6): 1483-1490.

[20] Gao H W, Petrosyan L A. Dynamics cooperative games. Beijing, Science Press, 2009. 